\title{
Phase transition in one dimensional model with unique ground state
}

\author{
Azer Kerimov ${ }^{1}$ \\ Department of Mathematics, Bilkent University, 06533 Bilkent, Ankara, Turkey \\ Received 2 June 1995; revised 1 November 1995
}

\begin{abstract}
A one-dimensional model having a unique ground state and admitting a phase transition is constructed.
\end{abstract}

Keywords: Hamiltonian; Ground state; Gibbs state; Markov chain

\section{The main result}

The problem of phase transitions in one-dimensional models is a field of interest of many authors. Examples of phase transitions are given in (all models but [1] are defined on $\left.\mathbf{Z}^{+}=\mathbf{Z}^{1} \cap[0, \infty)\right)$ [1] $(\Phi=1,-1$ and the interaction is of long range), [2,3] ( $\Phi$ is countable and the translation-invariant interaction is between nearest neighbors), $[4,5](\Phi=1,-1$ and the interaction is between nearest neighbors and non translationally invariant).

In this section we define a model (1) with a unique ground state having at least two limit Gibbs states. Consider a model on $\mathbf{Z}^{1}$ with the Hamiltonian

$$
H(\varphi(x))=\sum_{x \in Z^{1}} U_{x, x+1}^{1}(\varphi(x), \varphi(x+1))+U_{x}^{2}(\varphi(x))
$$

\footnotetext{
'E-mail: kerimov@fen.bilkent.edu.tr. Permanent address: Institute of Mathematics and Mechanics, Academy of Sciences of Azerbaijan, Baku 370602, Azerbaijan.
} 


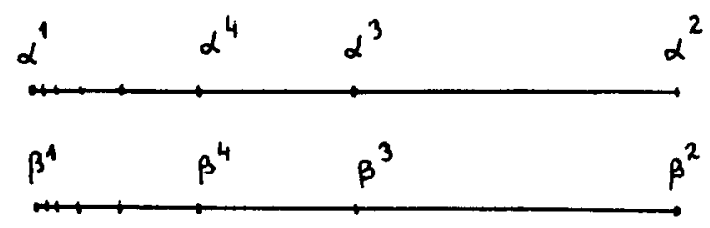

Fig. 1. Illustration of the distance function $\operatorname{dist}(.,$.$) .$

The spin space $\Phi$ of model (1) consists of a countable number of alpha spins $\alpha^{n}, n=$ $1,2, \ldots ;$ a countable number of beta spins $\beta^{n}, n=1,2, \ldots$; and a gamma spin $\gamma$. The metric in $\Phi$ is given by the following distance function dist(.,.) (see Fig. 1):

$$
\operatorname{dist}\left(\alpha^{m}, \alpha^{m+1}\right)=1 / 2^{m-1}, \quad \operatorname{dist}\left(\alpha^{m}, \alpha^{k}\right)=\sum_{l=m}^{l=k-1} \operatorname{dist}\left(\alpha^{l}, \alpha^{l+1}\right),
$$

for $k>m>1$,

$\operatorname{dist}\left(\alpha^{1}, \alpha^{2}\right)=1, \quad \operatorname{dist}\left(\alpha^{1}, \alpha^{m}\right)=1-\operatorname{dist}\left(\alpha^{2}, \alpha^{m}\right)$,

$\operatorname{dist}\left(\beta^{m}, \beta^{m+1}\right)=1 / 2^{m-1}, \quad \operatorname{dist}\left(\beta^{m}, \beta^{k}\right)=\sum_{l=m}^{l=k-1} \operatorname{dist}\left(\beta^{l}, \beta^{l+1}\right)$,

for $k>m>1$,

$\operatorname{dist}\left(\beta^{1}, \beta^{2}\right)=1, \quad \operatorname{dist}\left(\beta^{1}, \beta^{m}\right)=1-\operatorname{dist}\left(\beta^{2}, \beta^{m}\right)$,

$\operatorname{dist}\left(\alpha^{m}, \beta^{k}\right)=1, \quad \operatorname{dist}\left(\gamma, \alpha^{m}\right)=\operatorname{dist}\left(\gamma, \beta^{m}\right)=1, \quad$ for any $m, k$.

It can be readily verified that the function dist(.,.) defines a metric and the spin space $\Phi$ equipped with this metric is compact. Hence by Tikhonov's theorem the configuration spaces $\Phi_{V}=\prod_{i=-V}^{+V} \Phi$ and $\Phi_{\infty}=\prod_{i=-\infty}^{\infty} \Phi$ are compact.

The zero-interaction measure $\lambda$ on the space $\Phi$ is a counting measure [6].

Below we define the functions $U_{x, x+1}^{1}(\varphi(x), \varphi(x+1))$ and $U_{x}^{2}(\varphi(x))$. The first function $U_{x, x+1}^{1}(\varphi(x), \varphi(x+1))$ is bounded in any finite volume and the second function $U_{x}^{2}(\varphi(x))$ is not an interaction potential (it only controls the number of "admitted" spins, due to the $U^{2}$ in any finite volume the number of "admitted" configurations is finite). Thus, the set of all limit Gibbs states of the Hamiltonian (1) is not empty $[6,7]$.

The pair potential function of nearest neighbors $U_{x, x+1}^{1}(\varphi(x), \varphi(x+1))$ is symmetric with respect to the two arguments and symmetric with respect to the point $x=-1 / 2$. Thus,

$$
\begin{aligned}
& U_{x, x+1}^{1}\left(\varphi^{\prime}, \varphi^{\prime \prime}\right)=U_{x, x+1}^{1}\left(\varphi^{\prime \prime}, \varphi^{\prime}\right), \\
& U_{x, x+1}^{1}(\varphi(x), \varphi(x+1))=U_{-x-1,-x}^{1}(\varphi(-x-1), \varphi(-x)) .
\end{aligned}
$$

For nonnegative $x \in \mathbf{Z}^{1} U_{x, x+1}^{1}(\varphi(x), \varphi(x+1))$ is defined as:

$$
\begin{aligned}
& U_{x, x+1}^{1}\left(\alpha^{m}, \alpha^{k}\right)=1, \quad U_{x, x+1}^{1}\left(\beta^{m}, \beta^{k}\right)=1, U_{x, x+1}^{1}(\gamma, \gamma)=0, \\
& U_{x, x+1}^{1}\left(\alpha^{m}, \beta^{k}\right)=f_{x}, \quad U_{x, x+1}^{1}\left(\alpha^{m}, \gamma\right)=1, \quad U_{x, x+1}^{1}\left(\beta^{m}, \gamma\right)=1,
\end{aligned}
$$


where $m$ and $k$ are any natural numbers and

$$
f_{x}=-\ln \left\{\left[(4 / 3)^{1 / 2^{x}}-1\right] / 2\right\}+1 \text {. }
$$

The function $U_{x}^{2}(\varphi(x))$ playing the role of the external field is symmetric with respect to the point $x=1 / 2$. Thus, $U_{x-1}^{2}(\varphi(x-1))=U_{-x}^{2}(\varphi(-x))$ for $x>0$. For positive $x \in \mathbf{Z}^{1}, U_{x}^{2}(\varphi(x))$ is defined as:

$$
\begin{array}{ll}
U_{x}^{2}\left(\alpha^{m}\right)=U_{x}^{2}\left(\beta^{m}\right)=0, & \text { if } m \leq g_{x}, \\
U_{x}^{2}(\gamma)=0, & \\
U_{x}^{2}\left(\alpha^{m}\right)=U_{x}^{2}\left(\beta^{m}\right)=\infty, & \text { if } m>g_{x},
\end{array}
$$

where $m$ is any natural number and $g_{x}=2\left((4 / 3)^{1 / 2^{x}}-1\right)^{-1}$.

It can be readily verified that the configuration $\varphi(x)=\gamma, x \in \mathbf{Z}^{1}$ is the only ground state of the model (1). Let $I_{V}$ be the segment $[-V,+V]$. Suppose the boundary conditions $\varphi^{1}(x)=\varphi^{1}(x), x \in \mathbf{Z}^{1}-I_{V}$ are fixed and

$$
H_{V}\left(\varphi(x) \mid \varphi^{1}(x)\right)=\sum_{x=-V-1}^{V} U_{x, x+1}^{1}(\varphi(x), \varphi(x+1))+\sum_{x=-V}^{V} U_{x}^{2}(\varphi(x)) .
$$

Due to conditions (2) for any $V$ the number of "admitted" spin configurations is finite and the partition function

$$
\Xi_{V}=\sum_{\varphi(x) \in \Phi_{V}} \exp \left(-\beta H_{V}\left(\varphi(x) \mid \varphi^{1}(x)\right)\right)
$$

corresponding to the boundary conditions $\varphi^{1}(x), x \in \mathbf{Z}^{1}-I_{V}$ is finite.

In further calculations we restrict the value of the temperature by $T<1$, where $T=k T^{\prime}, T^{\prime}$ is the temperature, $k$ is the Boltzmann constant.

Theorem. Let $T<1$. There exist limit Gibbs states of model (1) $\mathbf{P}^{\alpha}$ and $\mathbf{P}^{\beta}$ such that

$$
\begin{aligned}
& \mathbf{P}^{\alpha}(\varphi(0)=\alpha)=\sum_{m=1}^{\infty} \mathbf{P}^{\alpha}\left(\varphi(0)=\alpha^{m}\right)>1 / 2, \\
& \mathbf{P}^{\beta}(\varphi(0)=\beta)=\sum_{m=1}^{\infty} \mathbf{P}^{\beta}\left(\varphi(0)=\beta^{m}\right)>1 / 2
\end{aligned}
$$

Proof. Due to the symmetry, we prove only the inequality

$$
\mathbf{P}^{\alpha}(\varphi(0)=\alpha)>1 / 2 \text {. }
$$

Thus, in order to prove the theorem it is sufficient to show that at any $V \mathbf{P}_{V}\left(\varphi(0)=\alpha \mid \varphi^{\alpha}\right) \geq 9 / 16$, where $\mathbf{P}_{V}\left(\varphi(x) \mid \varphi^{\alpha}\right)$ is the Gibbs distribution in the space $\Phi_{V}$ corresponding to the boundary conditions $\varphi^{\alpha}(x)=\alpha^{1}, x \in \mathbf{Z}^{1}-[-V, V]$.

Let $\mathbf{P}_{V}\left(\varphi(x)=\alpha, x \in[-V, V] \mid \varphi^{\alpha}\right)=\mathbf{P}_{V}\left(\bigcap_{x=-V}^{V} \bigcup_{m=1}^{\infty}\left(\varphi(x)=\alpha^{m} \mid \varphi^{\alpha}\right)\right.$. Obviously, $\mathbf{P}_{V}\left(\varphi(0)=\alpha \mid \varphi^{\alpha}\right) \geq \mathbf{P}_{V}\left(\varphi(x)=\alpha, x \in[-V, V] \mid \varphi^{\alpha}\right)$. 
In order to prove (3) we shall prove that

$$
\mathbf{P}_{V}\left(\varphi(x)=\alpha, x \in[-V, V] \mid \varphi^{\alpha}\right) \geq 9 / 16 .
$$

Define a Gibbs distribution $\mathbf{P}_{V}\left(\varphi(x) \mid \varphi^{\alpha, \text { left }}\right)$ in the space $\Phi_{V}$ corresponding to the boundary conditions $\varphi^{\alpha, \text { left }}(x)=\alpha^{1}, x \in(-\infty,-V-1]$ and $\varphi^{\alpha, \text { left }}(x)=\emptyset, x \in[V+$ $1, \infty)$. By definition

$$
\begin{aligned}
& \mathbf{P}_{V}\left(\varphi(x)=\alpha \mid \varphi^{\alpha}\right)=\frac{\sum_{\alpha} \exp \left(-1 / T\left(H\left(\varphi(x) \mid \varphi^{\alpha}\right)\right)\right)}{\sum \exp \left(-1 / T\left(H\left(\varphi(x) \mid \varphi^{\alpha}\right)\right)\right)} \\
& \mathbf{P}_{V}\left(\varphi(x)=\alpha \mid \varphi^{\alpha, \text { left }}\right)=\frac{\sum_{\alpha} \exp \left(-1 / T\left(H\left(\varphi(x) \mid \varphi^{\alpha, \text { left }}\right)\right)\right)}{\sum \exp \left(-1 / T\left(H \left(\varphi(x) \mid \varphi^{\alpha, \text { left })))}\right.\right.\right.}
\end{aligned}
$$

where the summations in both numerators are taken over all configurations $\varphi(x) \in \Phi_{V}$ such that $\varphi(x)=\alpha^{m}$ for some $m$ and both summations in the denominators are taken over all configurations $\varphi(x) \in \Phi_{V}$.

In model (1) "adjacent" spins (alpha, beta or gamma spins) tend to be aligned. That is, the Hamiltonian (1) can be interpreted as ferromagnetic. Thus, in the spirit of ferromagnetic inequalities the following lemma seems to be natural.

\section{Lemma 1.}

$$
\mathbf{P}_{V}\left(\varphi(x)=\alpha, x \in[-V, V] \mid \varphi^{\alpha, \text { left }}\right) \leq \mathbf{P}_{V}\left(\varphi(x)=\alpha, x \in[-V, V] \mid \varphi^{\alpha}\right) .
$$

Proof. Let us compare numerators and denominators of (5) and (6). Each term in the numerator of (5) is equal to $\exp (-1 / T)$ times the corresponding term of (6). Each term in the denominator of (5) is equal to $\exp (-1 / T)$ (respectively $\exp \left(-f_{x} / T\right)$ ) times the corresponding term of (6) if at $x=V \varphi(x)=\alpha^{m}$ for some $m$ or $\varphi(x)=\gamma$ (respectively $\varphi(x)=\beta^{m}$ for some $m$ ).

But $f_{x}>1$ for any nonnegative integer. Thus, the lemma is proved.

It follows from Lemma 1 that in order to prove the theorem it is sufficient to establish the following inequality.

\section{Lemma 2.}

$$
\mathbf{P}_{V}\left(\varphi(x)=\alpha, x \in[-V, V] \mid \varphi^{\alpha, \text { left }}\right) \geq 9 / 16
$$

Proof. Consider a Markov chain (nonhomogeneous) starting at point $x=-V$ and ending at point $x=V$ with initial condition $\varphi(-V-1)=\alpha^{1}$ with transition probabilities $\pi_{\xi(x), \xi(x+1)}\left(\pi_{\xi(x), \xi(x+1)}\right.$ is the probability of the event that $\varphi(x+1)=\xi(x+1)$ on condition that $\varphi(x)=\xi(x))$, where

$$
\pi_{\xi(x), \xi(x+1)}=\mathbf{P}_{V}(\varphi(x+1)=\xi(x+1) \mid \varphi(x)=\xi(x), \varphi(x+2)=\emptyset) .
$$

Condition (2) implies that this Markov chain in $[-V, V]$ can be treated as a Markov chain with finite spin space. It follows from the definitions that 


$$
\mathbf{P}_{V}\left(\varphi(x)=\xi(x), x \in[-V, V] \mid \varphi^{\alpha, \text { left }}\right)=\pi_{\xi(-V-1)=\alpha^{1}, \xi(-V)} \prod_{x=-V}^{V-1} \pi_{\xi(x), \xi(x+1)}
$$

Define $\pi_{\xi(x)=\alpha, \xi(x+1)=\alpha}=\sum \pi_{\xi(x)=\alpha^{m}, \xi(x+1)=\alpha^{k}}$, where the summation is taken over all possible values of $k$ [by definition, the sum consists of a finite number of terms (due to (2)) and does not depend on $m]$. Thus,

$$
\begin{aligned}
\mathbf{P}_{V}(\varphi(x) & \left.=\alpha, x \in[-V, V] \mid \varphi^{\alpha, \text { left }}\right)=\pi_{\xi(-V-1)=\alpha^{1}, \xi(V)=\alpha} \prod_{x=-V}^{V-1} \pi_{\xi(x)=\alpha, \xi(x+1)=\alpha} \\
& =\prod_{x=-V-1}^{V-1} \pi_{\xi(x)=\alpha, \xi(x+1)=\alpha .}
\end{aligned}
$$

By definition $(T \leq 1)$,

$$
\begin{aligned}
& \pi_{\xi(x)=\alpha, \xi(x+1)=\alpha} \\
& =\frac{\sum_{i=1}^{g(x+1)} \exp (-1 / T)}{\sum_{i=1}^{g(x+1)} \exp (-1 / T)+\sum_{i=1}^{g(x+1)} \exp (-f(x+1) / T)+\exp (-1 / T)} \\
& =\frac{g(x+1) \exp (-1 / T)}{g(x+1) \exp (-1 / T)+g(x+1) \exp (-f(x+1) / T)+\exp (-1 / T)} \\
& =[1+\exp (-1 / T(f(x+1)-1))+1 / g(x+1)]^{-1} \\
& \geq[1+\exp (-f(x+1)+1)+1 / g(x+1)]^{-1} \geq(3 / 4)^{1 / 2^{x+1}} .
\end{aligned}
$$

Now note that

$$
\mathbf{P}_{V}\left(\varphi(x)=\alpha, x \in[-V, V] \mid \varphi^{\alpha, \text { left }}\right) \geq\left(\prod_{x=0}^{\infty} \pi_{\xi(x)=\alpha, \xi(x+1)=\alpha}\right)^{2}
$$

Thus, in order to prove (7) it is enough to show that

$$
\prod_{x=0}^{\infty} \pi_{\xi(x)=\alpha, \xi(x+1)=\alpha} \geq 3 / 4
$$

But

$$
\prod_{x=0}^{\infty} \pi_{\xi(x)=\alpha, \xi(x+1)=\alpha} \geq \prod_{x=1}^{\infty}(3 / 4)^{1 / 2^{x}}=3 / 4
$$

Thus, inequality (8), and hence Lemma 2 is proved.

Now inequality (4) is a direct implication of Lemma 2 and Lemma 1. Consider a sequence of probability distributions $\mathbf{P}_{V}\left(\varphi(x) \mid \varphi^{\alpha}\right)$. This sequence consists of at least one limit point, and this limit point $\mathbf{P}^{\alpha}$ is a limit Gibbs state [6,7]. Now Theorem 2 follows from inequality (3). 


\section{Conclusions}

In Ref. [8] the absence of phase transitions in one-dimensional models with longrange antiferromagnetic potentials is proved. Based on the methods of Ref. [8] we formulate the following:

Conjecture. Any one-dimensional model with discrete (at most countable) spin space and with a unique ground state has a unique Gibbs state if the spin space of this model is finite or the potential of this model is translationally invariant.

There exists a model with a unique ground state, bounded coupling potential and admitting phase transition (the interaction in model (1) is not bounded), such that it has a countable spin space, inhomogeneous external field and long-range ferromagnetic potential as in Ref. [1].

\section{Acknowledgement}

The author thanks the referee for his or her suggestions.

\section{References}

[1] F.J. Dyson, Commun. Math. Phys. 12 (1969) 91-107.

|2| F. Spitzer, J. Func. Anal. 20 (1975) 240-255.

[3] S. Kalikow, Ann. Prob. 5 (1977) 467-469.

[4] W.G. Sullivan, Commun. Dublin Inst. for Advanced Studies (1975).

[5] M.J. Miyamoto, Math. Kyoto Univ. 24 (4) (1984) 679-688.

[6] H.O. Georgii, Gibbs Measures and Phase Transitions (De Gruyter, Berlin, New York, 1988).

[7] Ya.G. Sinai, Theory of Phase Transitions, Rigorous Results, (Acad. Kiado, Budapest, 1982).

|8| A.A. Kerimov, J. Stat. Phys. 72 (3/4) (1993) 571-620. 